\title{
Research on Solid-phase Grinding Method of Manufacturing One-dimensional Zinc Oxide Nanomaterials Based on Nanosensors
}

\author{
Qiang Zou ${ }^{1, a}$, Shentong Mo,b, Guohui $\mathrm{Li}^{2, c, \text {, }}$ and Bing $\mathrm{Yu}^{\text {3,4,d }}$ \\ ${ }^{1}$ School of Mircoelectronics, Tianjin University, Weijin Road, Tianjin, China \\ ${ }^{2}$ Tianjin Development Zone Aojin High New Technology co., LTD, Weijin Road, Tianjin, China \\ ${ }^{3}$ School of Environmental Science and Engineering, \\ Tianjin University, Weijin Road, Tianjin, China \\ ${ }^{4}$ Shanghai Yanhua Smartech Group, Shanghai, China \\ a moshentong678@126.com, ${ }^{\mathrm{b}}$ zouqiang@tju.edu.cn, ${ }^{\mathrm{c}}$ liguohui@tju.edu.cn, ${ }^{\mathrm{d}}$ yubing@tju.edu.cn \\ *corresponding author
}

Keywords: Nanomaterials, Solid State Grinding Method, Zinc oxide(ZnO), One-dimension, Scanning Electron Microscope (SEM)

Abstract. In this paper, a new process for the preparation of one-dimensional $\mathrm{ZnO}$ nanomaterials was studied. A single-dimensional $\mathrm{ZnO}$ nanowire was synthesized by solidphase grinding method. The SEM images were taken first and then the SEM images of the products were compared in different ways. In order to understand the mechanism of product formation better, the surface morphology and composition of the products were observed. The optimum conditions for the preparation of one-dimensional $\mathrm{ZnO}$ nanowires were established, which provided important reference value for the production of nanomaterials. Besides, the solid-phase grinding method, in a large scale, would exert an unquestionably positive impact on manufacturing nanomaterials, as well as render the preparation more effective.

\section{Introduction}

Nanomaterials are materials that are at least one dimension in a three-dimensional space and they are in the nanoscale range (1 to $100 \mathrm{~nm}$ ). Nanomaterials are applied as a base unit because they have electrical and magnetic properties, which are distinctly different from those of conventional bulk materials, such as chemical, mechanical and other physical properties [1-2]. The nanostructured materials have large specific surface area, surface atomic number, surface energy and surface tension increase with the particle size of the descending hormone. Owing to manifesting in the surface effect, volume effect and quantum size effect characteristics, the nanoparticles are different from normal particles in heat, sensitive properties and surface stability, which makes the nanostructure material has a broad application prospects [3-5]. 
$\mathrm{ZnO}$ is a direct band gap II-VI compound, belonging to the N-type conductivity of the degenerate semiconductor material. Nano- $\mathrm{ZnO}$, as an excellent semiconductor oxide material, shows other materials unparalleled superior performance in the photoelectric, chemical aspects. The aspects mainly consist of significant quantum confinement effect, strong Ultraviolet (UV) absorption, low threshold high efficiency photoelectric properties, UV laser emission, piezoelectric and photocatalytic and carrier transportation, etc. In addition, $\mathrm{ZnO}$ nanostructures exhibit piezoelectric properties. Consequently, quasi-one-dimensional nanobelt structures have potential applications in micro-nanosensors, such as resonators, converters, and suspended bridges [6-8]. Besides, since $\mathrm{ZnO}$ has the advantages of negative electron affinity, high mechanical strength, high thermal stability, oxidation resistance and large exciton energy, it is superior to field emission.

Recently, the researchers had different methods to prepare the structure and morphology of different $\mathrm{ZnO}$ nanostructures, which were the largest nano-structure of semiconductor materials [9]. In 1997, Jiafu Zhong heated Zn-Fe-C-O powder by thermal oxidation method to prepare high-yield hollow four-pin $\mathrm{ZnO}$ nanostructures [10-11]. In 2001, the international authority of the magazine 'Science' has reported Z.W.Pan from the United States Georgia Institute of Technology and others, who obtained $\mathrm{ZnO}$ and other semiconductor oxide nanobelt by the high temperature evaporation of oxide powder method. In addition, M.H.Huang, from the University of California, observed the UV stimulated emission of $\mathrm{ZnO}$ nanowire arrays at room temperature, which led to great concern by the scientific and technological community [12-13]. In 2008, Kehua Zhang, in the absence of catalyst, directly heated zinc oxide tablets in the air, as well as successfully prepared $\mathrm{ZnO}$ nanowires and nanoflakes [14-15]. In 2009, Yue Yang prepared the $\mathrm{ZnO}$ thin films by directly heating oxidized magnetron sputtering deposited metal zinc thin films. The preparation parameters include oxidation temperature and holding time [16-17]. The synthesis characteristics of zinc oxide thin films were obtained by systematic study of the process parameters. Moreover, the structural characteristics and properties of the zinc oxide films synthesized in this work were revealed. They established the relationship between the synthesis process and the structure or properties of the films. The structure of the films was analysed by X-ray diffraction, Raman spectroscopy and SEM. The luminescent properties of the films were studied by photoluminescence method. In 2011, Su Li prepared $\mathrm{ZnO}$ one-dimensional nanostructures by non-catalyst thermal evaporation method. The effects of preparation parameters and additives on the growth were studied. The growth mechanism of $\mathrm{ZnO}$ was studied. Based on this, they researched the $\mathrm{ZnO}$ one-dimensional nanostructures and the optical properties of the material. It can be seen that the research of $\mathrm{ZnO}$ nanomaterials has become a new frontier issue [18-20].

There are many methods for preparing nano-ZnO materials. The purity of the powdery material prepared by physical method is high, but the cost of equipment is large and the yield is small. The yield produced by chemical method is large, but it is usually produce by calcining or drying, which makes the product contain some impurities [21-22]. Therefore, in the preparation of nano- $\mathrm{ZnO}$ materials should be combined with its use requirements and the appropriate preparation method. The preparation method of nano- $\mathrm{ZnO}$ material is still in the stage of theoretical exploration and experiment, which has not yet reached the scale of industrialization. Besides, its extensive industrial application is greatly restricted. Therefore, the formation mechanism and microstructure of nano$\mathrm{ZnO}$ and the preparation method of high purity nano- $\mathrm{ZnO}$ need further study. In this experiment, a series of one-dimensional $\mathrm{ZnO}$ nanomaterials were prepared by solid-phase grinding method. The surface morphology of the products was analysed by SEM, and the best preparation scheme was selected to facilitate the industrialization of nanomaterials. 


\section{Experiment}

Firstly, the precursors were prepared by the reaction of zinc nitrate hexahydrate, as raw material, and solid-phase sodium hydroxide $(\mathrm{NaOH})$. Then, mixing the precursors with sodium chloride $(\mathrm{NaCl})$ in different proportions. The nanoparticles were regenerated under high temperature condition, as well as the oxide nanorods were obtained.

The specific experimental method is as follows. $\mathrm{Zn}\left(\mathrm{NO}_{3}\right)_{2}$ and $\mathrm{NaOH}$ were weighed at a molar ratio of 1: 2 , and thoroughly grinded in the mortar. The system released a lot of heat, quickly absorbed moisture and became wet white milk. As the grinding time passes, the system became dry white powder and has aggregated lumps formed. After $30 \mathrm{~min}$, the resulting white product was washed three times with distilled water and then filtered three times with anhydrous ethanol to obtain a washed white powder. Drying continued at constant temperature. And the resulting white powder was actually the $\mathrm{ZnO}$ precursor. The molar ratio of precursor and $\mathrm{NaCl}$ was 1: 2 or 1: 4 . Two molar ratios were obtained, which were thoroughly grinded for $30 \mathrm{~min}$. There was no heat and moisture absorption during the grinding process. In order to produce a yellowish product, the system after grinding and the simple precursors without $\mathrm{NaCl}$ were calcined at a constant temperature of $400{ }^{\circ} \mathrm{C}$ in a muffle furnace. Then, the product above was washed three times with distilled water and washed three times with absolute ethanol. Finally, the product was obtained by drying the system.

\section{Results and discussion}

The SEM images of the samples obtained from the different schemes which were designed for different ratios of precursors and $\mathrm{NaCl}$ in the experiment are shown below.

Table 1 Preparation of $\mathrm{ZnO}$ nanowires by solid-phase grinding method

\begin{tabular}{|c|c|c|c|c|c|c|}
\hline Heating time & $60 \mathrm{~min}$ & $30 \mathrm{~min}$ & $60 \mathrm{~min}$ & $120 \mathrm{~min}$ & $30 \mathrm{~min}$ & $60 \mathrm{~min}$ \\
\hline $\begin{array}{c}\text { The molar ratio } \\
\text { of precursor and } \\
\mathrm{NaCl}\end{array}$ & $\begin{array}{c}\text { No NaCl } \\
\text { participation }\end{array}$ & $1: 2$ & $1: 2$ & $1: 2$ & $1: 4$ & $1: 4$ \\
\hline SEM images & Figure 1-A & $\begin{array}{c}\text { Figure 1- } \\
\mathrm{B}\end{array}$ & $\begin{array}{c}\text { Figure 1- } \\
\mathrm{C}\end{array}$ & $\begin{array}{c}\text { Figure 1- } \\
\mathrm{D}\end{array}$ & Figure 1-E & Figure 1-F \\
\hline
\end{tabular}



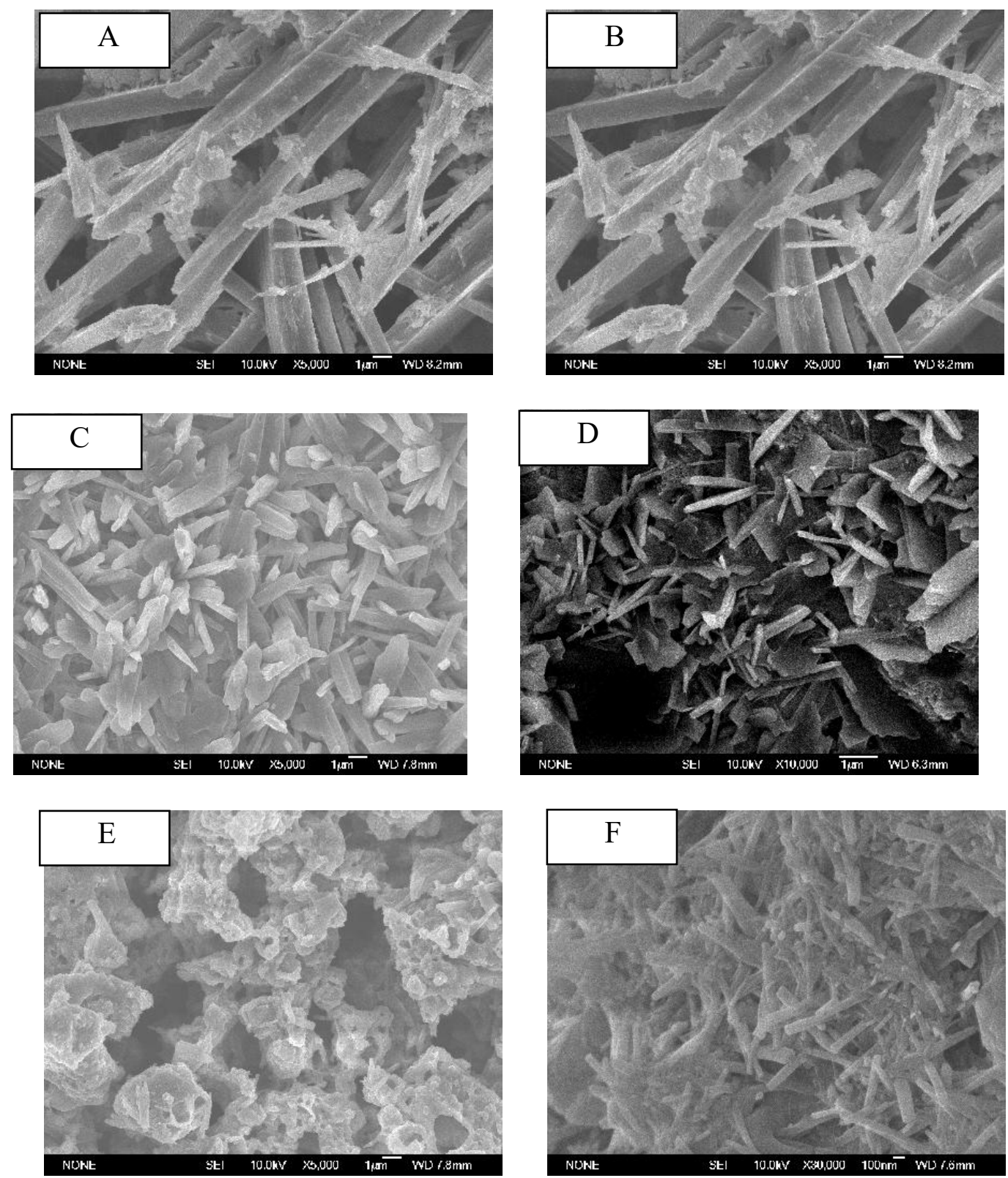

Figure $1 \mathrm{SEM}$ image of the sample (A. no $\mathrm{NaCl}$ involved in the reaction, heated for $60 \mathrm{~min}$;

B. precursors and $\mathrm{NaCl}$ were heated at 1:2 for $30 \mathrm{~min}$;

C. precursors and $\mathrm{NaCl}$ were heated at 1: 2 for $60 \mathrm{~min}$;

D. precursor and $\mathrm{NaCl}$ were heated at 1: 2 for $120 \mathrm{~min}$;

E. precursors and $\mathrm{NaCl}$ were heated at 1: 4 for $30 \mathrm{~min}$;

F. precursors and $\mathrm{NaCl}$ were heated at 1: 4 for $60 \mathrm{~min}$ )

\subsection{Effect of $\mathrm{NaCl}$ on the particle size of nano- $\mathrm{ZnO}$}

In comparison with Figure 1-A and Figure 1-C, it can be found that when nano-ZnO is prepared with $\mathrm{Zn}\left(\mathrm{NO}_{3}\right)_{2}$ and $\mathrm{NaOH}$, the particle size of the nanorods obtained by calcination with $\mathrm{NaCl}$ and the precursor is larger than that of $\mathrm{NaCl}$-free reaction. And the particle size in $\mathrm{NaCl}$-free reaction is about $50 \mathrm{~nm}$ to $150 \mathrm{~nm}$. The particle size obtained by adding $\mathrm{NaCl}$ is between $100 \mathrm{~nm}$ and $150 \mathrm{~nm}$. By observing the synthetic nanostructure, it is easy to form a rod-shaped nanostructures with more 
regular shape in $\mathrm{NaCl}$ participation. But without $\mathrm{NaCl}$ participation, the nanostructure morphology is not very regular, such as linear, flaky and columnar, etc.

\subsection{The optimum molar ratio of $\mathrm{NaCl}$ and the precursor}

$\mathrm{Zn}\left(\mathrm{NO}_{3}\right)_{2}$ and $\mathrm{NaOH}$ (molar ratio of 1: 2) were prepared by mixing the precursor with $\mathrm{NaCl}$ at a molar ratio of 1: 2,1: 4 to prepare the $\mathrm{ZnO}$ nanostructures. The protons under the same temperature condition were observed by SEM as shown in Figure 1-B and Figure 1-E, Figure 1-C and Figure 1$\mathrm{F}$. The former diameter of product heated for $30 \mathrm{~min}$ is about $100 \mathrm{~nm}$, while the latter for $60 \mathrm{~min}$ is greater than $200 \mathrm{~nm}$. When the molar ratio of precursor and $\mathrm{NaCl}$ is $1: 2$ at the same temperature and calcination time, the particle size of the nanorods produced by the other molar ratio is stable and the reaction is comprehensive. Hence, the best molar ratio of the precursor and $\mathrm{NaCl}$ is $1: 2$.

\subsection{Time factors for the growth of $\mathrm{ZnO}$ nanowires}

The precursors and $\mathrm{NaCl}$ were calcined at the molar ratio of 1: 2 and heated in a muffle furnace. And the SEM images was observed for the different heat treatment time. It can be seen that with the increase in heat treatment time, the resulting nanostructure after grinding the fixed ratio precursor and $\mathrm{NaCl}$ is more rod-like and longer. In addition, the product for $60 \mathrm{~min}$ is more uniform and has smaller particle size. Thus, when the molar ratio of precursors and $\mathrm{NaCl}$ is $1: 2$, the $\mathrm{ZnO}$ onedimensional nanostructures obtained by heating for $60 \mathrm{~min}$ are more ideal.

\subsection{Temperature factors for the growth of $\mathrm{ZnO}$ nanowires}

$\mathrm{Zn}\left(\mathrm{NO}_{3}\right)_{2}$ and $\mathrm{NaOH}$ were subjected to solid-phase grinding at a molar ratio of 1: 2, washed successively with distilled water and absolute ethanol. And then they were filtered and placed in a muffle furnace for heat treatment at a temperature of $350{ }^{\circ} \mathrm{C}$ for one hour. And the product was obtained as shown in Figure 3. Based on the fixed precursor, the experiment can determine the temperature factor on the growth of nanorods by changing the experimental temperature. It can be seen that the product obtained by heating at a temperature of $350{ }^{\circ} \mathrm{C}$ has reached a nanoscale level but has not yet formed a one-dimensional structure, when the particle size of the product obtained by heating at $350{ }^{\circ} \mathrm{C}$ for 1 hour is compared with that at $400{ }^{\circ} \mathrm{C}$ for 1 hour. And the product is still in the state of particles, while the length of it is much smaller than that of the product obtained at $400{ }^{\circ} \mathrm{C}$. Therefore, it can be determined that the preparation of $\mathrm{ZnO}$ one-dimensional nanostructure temperature should be at least greater than or equal to $400{ }^{\circ} \mathrm{C}$, so as to grow one-dimensional structure of a nanoscale level. 


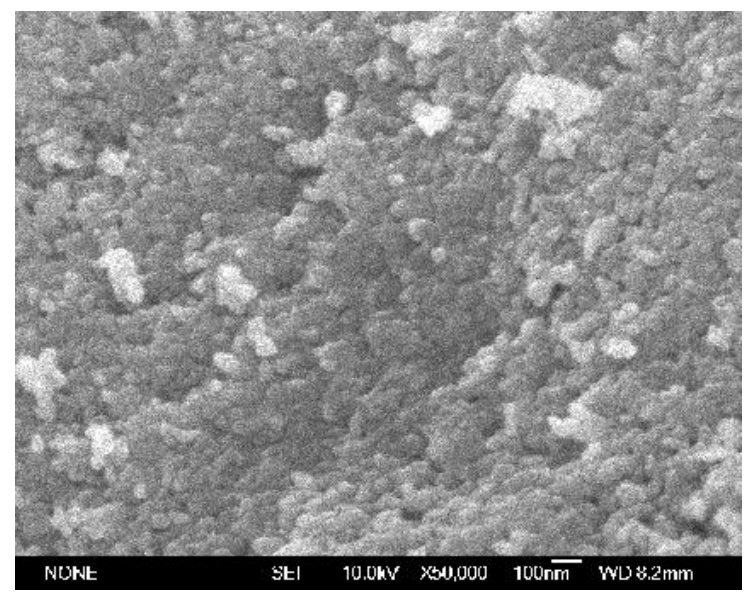

Figure $2 \mathrm{Zn}\left(\mathrm{NO}_{3}\right)_{2}$ and $\mathrm{NaOH}$ were subjected to solid-phase grinding at a molar ratio of 1:2 and then heated at $350{ }^{\circ} \mathrm{C}$ for $60 \mathrm{~min}$

\subsection{The effect of raw materials on the formation of precursors}

The zinc acetate and $\mathrm{NaOH}$ were subjected to solid-phase grinding at a molar ratio of 1: 2 , then washed successively with distilled water and absolute ethanol, and the precursor was obtained by filtration. The precursor and $\mathrm{NaCl}$ were calcined at a molar ratio of 1: 2, and placed in a muffle furnace for heat treatment at $400{ }^{\circ} \mathrm{C}$ for one hour. The resulting product was washed three times with distilled water and three times with anhydrous ethanol. The SEM image of resulting product was shown in Figure 3.

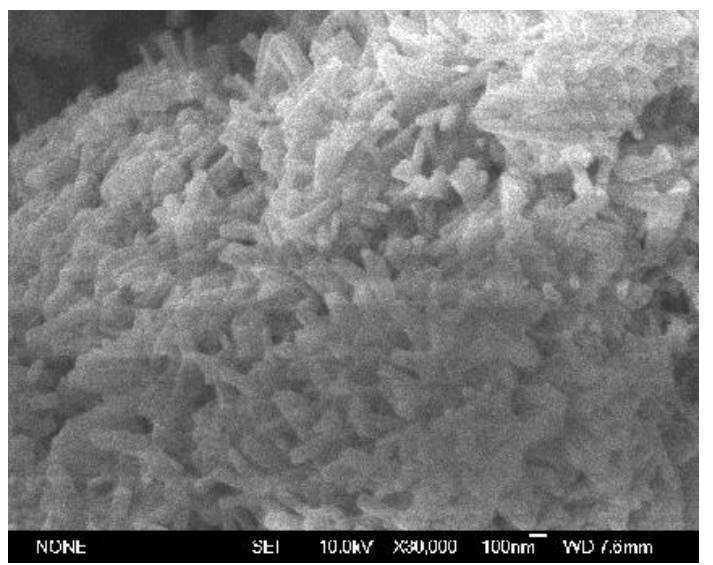

Figure 3 Zinc acetate and $\mathrm{NaOH}$ were subjected to solid-phase grinding at a molar ratio of 1:2 and then heated at $400{ }^{\circ} \mathrm{C}$ for $60 \mathrm{~min}$

Compared with Figure 4 and Figure 2-C, the particle size of products is both about $100 \mathrm{~nm}$, and the shape and length of products are relatively uniform and similar. It can be concluded that the anions in the metal salts containing the $\mathrm{Zn}$ elements, as the raw materials, required for the preparation of the $\mathrm{ZnO}$ precursors have little effect on the formation of the $\mathrm{ZnO}$ precursors. And the precursor factor need not be taken into account when preparing the precursor.

\subsection{Mechanism Analysis of Solid-phase Synthesis}

$\mathrm{NaCl}$, as a mineralizer, plays a role of the molten salt medium in the process of the preparation. The purpose of mineralizing agent is to change the physical and chemical conditions of crystal growth, such as molten salt species, sintering conditions and the ratio of molten salt. In addition, it 
used to promote or control the formation of crystalline compounds or reaction. Because there are differences in the stability of the oxide growth primers with different structural forms and dimensions, by changing the structure of the growth of the lava in the structure, the rate at which they are superimposed on the crystal face is different, resulting in a difference in the growth rate of each crystal face. Thus, the control of the crystal morphology ultimately determines the structure, shape and size of the crystal.

In this molten salt medium method, the salt anion should play the main role. The chloride ions in the molten state have a certain coordination capacity. Consequently, it is easy to form a coordination bond with $\mathrm{ZnO}$ cation of a nanoscale level, and chloride ions to form a coordination bond crystal face. Due to the presence of chloride ions to inhibit the growth of their own crystals, and other crystal surface relatively faster growth rate, the growth of $\mathrm{ZnO}$ has obvious anisotropy, and the product molecules are arranged in an orderly rod-like structure. The metal oxide onedimensional nanomaterials were prepared in the end.

Molten salt medium $\mathrm{NaCl}$ is a key factor in the self-growth of nanoparticle precursors. In addition to the role of coordination bond, high temperature $\mathrm{NaCl}$ salt also has the ability to dissolve $\mathrm{ZnO}$ nanoparticles; its molten salt ion low viscosity and rapid diffusion are conducive to the high rate of mass transfer, heat transfer or energy transfer rate and high efficiency during the process of nanoparticle recrystallization. At the same time, the $\mathrm{NaCl}$ has good thermal stability and provides a stable liquid environment for the self-assembling growth of nanoparticles. Therefore, the solidphase reaction method at room temperature only provides nano-scale nanoparticle precursors. The key to self-growth of nanoparticles is the second step, the application of molten salt technology in the annealing stage.

The morphological transition of one-dimensional $\mathrm{ZnO}$ nanorods, that is, the transition from zerodimensional nanoparticles to one-dimensional nanorods is as follows. The $\mathrm{ZnO}$ nanoparticle precursors prepared by solid-phase reaction at room temperature are calcined. The nanoparticle precursors are rearranged under the action of surface chloride ion coordination bonds. When the temperature rises to a certain value, the salt melts to form the melt. Owing to the low viscosity of the molten salt and good fluid flow, the precursor $\mathrm{ZnO}$ nanoparticles rapidly diffuse, rearrange and grow in the rapidly flowing melt, finally resulting in forming the nanorods.

The diameter and length of the nanorods are increasing as the firing time increases or the temperature increases. It is shown that the $\mathrm{ZnO}$ nanoparticles can self-grow to form nanorods, onedimensional nanomaterials, under the condition of molten salt at a certain temperature. As shown in Figure 4, this is a transition process of the same material from one solid-phase to another solidphase.

molten salt medium

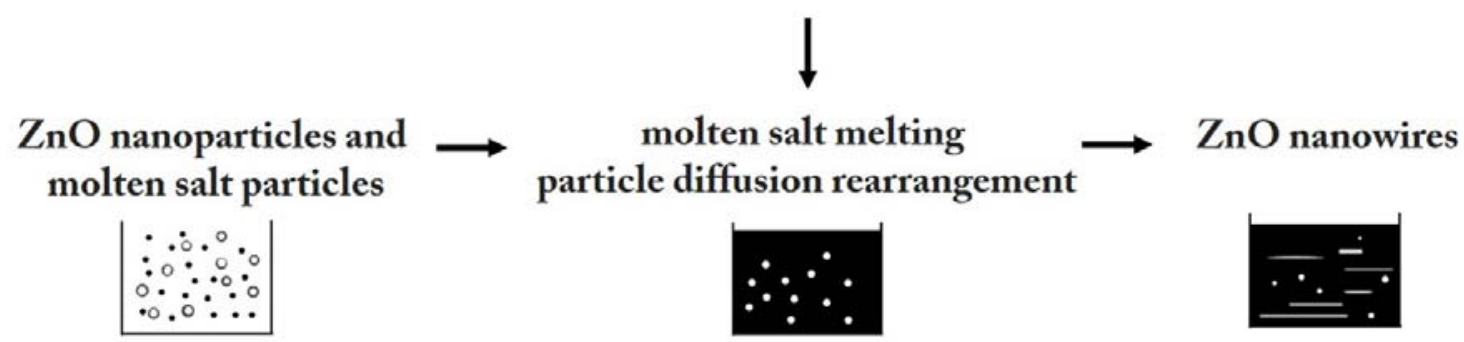

Figure 4 Process of solid phase transition

It is obvious that the solid-phase transformation mechanism of $\mathrm{ZnO}$ nanorods in molten salt has both the characteristics of self-assembling growth mechanism and the growth mechanism of solution based on covering agent. And the characteristics of solid-phase transformation mechanism 
is also similar to the characteristics of solid-liquid-solid (SLS) mechanism. However, it is clear that the growth mechanism of this solid-phase transformation is different from those growth mechanisms mentioned above. And the main feature is that the solid-phase transition of the same material in different nano-scale form, which occurs in the dissolved salt medium. The solid growth mechanism of $\mathrm{ZnO}$ nanorods is a new growth mechanism.

\section{Conclusions}

In summary, a new process for the preparation of $\mathrm{ZnO}$ nanorods was studied in this paper. Firstly, the $\mathrm{ZnO}$ nanoparticle precursors were prepared by solid-phase reaction at room temperature. Then, in the molten salt medium, the nanoparticle precursor was diffused to render the crystal grow again. The $\mathrm{ZnO}$ nanorods, one-dimensional nanostructured materials were prepared in the end. The morphology of $\mathrm{ZnO}$ nanorods was analysed by SEM. The diameter of $\mathrm{ZnO}$ nanorods was about 80 $\mathrm{nm}$ to $100 \mathrm{~nm}$, and the length was from nano-scale to micro-scale. In the growth process of $\mathrm{ZnO}$ nanorods, only in the use of molten salt medium conditions, $\mathrm{ZnO}$ nanoparticles can be self-grown to form nanorods. Molybdenum salt plays a key role in self-growing one-dimensional nanostructures.

The formation process of $\mathrm{ZnO}$ nanorods is a process of solid-phase transformation growth, which is a new growth mechanism of one-dimensional nanomaterials. In this paper, $\mathrm{Zn}\left(\mathrm{NO}_{3}\right)_{2}$ was reacted with $\mathrm{NaOH}$ to prepare nano-ZnO. The particle size of the nano- $\mathrm{ZnO}$ prepared by the addition of $\mathrm{NaCl}$ in the precursor was larger and more uniform than that of the $\mathrm{ZnO}$. Chloride ion has an effect on promoting the growth of nanosized particles in the preparation of nano- $\mathrm{ZnO}$ reaction. The optimum molar ratio of precursor and $\mathrm{NaCl}$ is $1: 2$. The morphology of nano- $\mathrm{ZnO}$ prepared with chloride ion is more likely to form a rod-like structure, and the morphology of $\mathrm{ZnO}$ nanorods without chloride ions is generally flaky. The change in heat treatment time and temperature exert a certain impact on the growth of $\mathrm{ZnO}$ nanorods. Time is 60 minutes for the best and the heating temperature should be above $400{ }^{\circ} \mathrm{C}$, so that the one-dimensional nanostructures of $\mathrm{ZnO}$ can be formed. The change of metal salt raw materials has little effect on the growth of $\mathrm{ZnO}$ nanorods. In addition, the metal salts containing zinc ions can be generally used to prepare $\mathrm{ZnO}$ precursor.

\section{Acknowledgements}

This work was supported by Ministry of Science and Technology of the People's Republic of China (Contract No. 2015BAJ01B01), as well as sponsored by Center for post-doctoral studies in Tianjin Development Zone Aojin High New Technology co., LTD.

\section{References}

[1] King S L, Gardeniers J G E and Boyd I W. (1996) Pulsedlaser Deposited ZnO for Device Application [J]. Appl. Surf. Sci, 96(96), 811-818.

[2] H Kind, H Yan, B Messer, M Law and P Yang. (2002) Nanowire Ultraviolet Photodetectors and Optical Switches [J]. Adv. Mater, 14(2), 158-160.

[3] Y He, W Sang, J Wang, R Wu and J Min. (2005) Polymer-assisted Complexing Controlled Orientation Growth of ZnO Nanorods [J]. Nanopart. Res, 7(2), 307-311.

[4] Bagnall D M, Chen Y F, Zhu Z, Tianxue Yao, S Koyama, Miaosen Shen and Takenari Goto. (1997) Optically Pumped Lasing of $\mathrm{ZnO}$ at Room Temperature [J]. App. Phys. Lett, 70(17), 2230-2232.

[5] Matthieu Chollet, Laurent Guerin, Naoki Uchida, Souichi Fukaya, Hiroaki Shimoda, Tadahiko Ishikawa, Kazunari Matsuda, Takumi Hasegawa, Akira Ota, Hideki Yamochi, Gunzi Saito, Ryoko Tazaki, Shin-Ichi Adachi 
and Shin-Ya Koshihara. (2005) Gigantic Photoresponse in 1/4-filled-band Organic Salt (EDO-TTF)2PF6 [J]. Science, 307(5706), 86-89.

[6] SA Morin, MJ Bierman, J Tong and S Jin. (2010) Mechanism and Kinetics of Spontaneous Nanotube Growth Driven by Screw Dislocations [J]. Science, 328(5977), 476-480.

[7] BJ Jin, SH Bae, SY Lee and S Im. (2000) Effects of Native Defects on Optical and Electrical Properties of ZnO Prepared by Pulsed Laser Deposition [J]. Mater. Sci. Eng B, 71(1), 301-305.

[8] O. Dulub, L. A. Boatner and U. Diebold. (2002) STM Study of the Geometric and Electronic Structure of

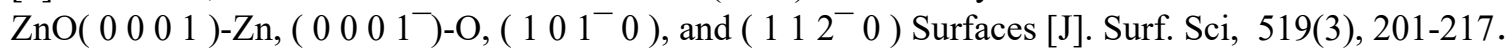

[9] B. Meyer and D. Marx. (2003) Density-functional Study of the Structure and Stability of ZnO Surfaces [J]. Phys. Rev, 67(3), 039902.

[10] Wang M, Fei G T and Zhang L D. Porous-ZnO-nanobelt Film as Recyclable Photocatalysts with Enhanced Photocatalytic Activity [J]. Nanoscale Res Lett, 2010, 5(11), 1800-1803.

[11] Srikantv and Clarke D R. (1998) On the Optical Band Gap of Zinc Oxide [J]. Appl. Phys, 83(10), 54475451 .

[12] Y J Xing, Z. H. Xi, Z. Z. Q. Xue, X. D. Zhang, J. H. Song, Rongming Wang, Jinzhang Xu, Y. Song, Shu-Lin Zhang and Dapeng Yu. (2003) Optical Properties of the ZnO Nanotubes Synthesized via Vapor Phase Growth [J]. Appl. Phys. Lett, 83(9), 1689-1692.

[13] Wang Z and Li H L. (2002) Highly Ordered Zinc Oxide Nanotubules Synthesized within the Znodic Aluminum Oxide Template [J]. Appl. Phys, 74(2), 93-94.

[14] Yongshu Tian, Chenguo Hu, Yufeng Xiong, Buyong Wan, Chuanhui Xia, Xiaoshan He and Hong Liu. (2010) ZnO Pyramidal Arrays: Novel Functionality in Antireflection [J]. J. Phys. Chem. C, 114(22), 10265-10269.

[15] Z W Pan, Z R Dai and Z L Wang. (2001) Nanobelts of Semiconducting Oxides [J]. Science, 291(5510):19471949.

[16] Hongxia Li, Jiyang Wang, Hong Liu, Changhong Yang, Hongyan Xu, Xia Li and Hongmei Cui. (2004) Solgel Preparation of Transparent Zinc Oxide Films with Highly Preferential Crystal Orientation [J]. Vacuum, 77(1), 57-62.

[17] Zhou Gui, Jian Liu, Zhengzhou Wang, Lei Song, Yuan Hu, Weicheng Fan and Daoyong Chen. (2005) From Muticomponent Precursor to Nanoparticle Nanoribbons of ZnO [J]. Phys. Chem, 109(3), 1113-1117.

[18] Li S Y, Lee C Y and Tseng T Y. (2003) Copper-catalyzed ZnO Nanowires on Silicon (100) Grown by Vapor -liquid-solid Process [J]. Journal of Crystal Growth, 247(3-4), 357-362

[19] Dapeng Yu, Y.J Xing, Q.L Hang, Hanfei Yan, Jinzhang Xu, Z.H Xi and Shi-Qing Feng. (2001) Controlled Growth of Oriented Amorphous Silicon Nanowires via a Solid-liquid-solid(SLS) Mechanism [J]. Physics E, 9(2), 305-309.

[20] B. Q. Cao, J. Zúñiga-Pérez, C. Czekalla, H. Hilmer, J. Lenzner, N. Boukos, A. Travlos, M. Lorenz and M. Grundmann. (2010) Tuning the Lateral Density of ZnO Nanowire Arrays and its Application as Physical Templates for Radial Nanowire Heterostructures. J. Mater. Chem, 20(19), 3848-3854.

[21] YB Li, Y Bando, D Golberg and K Kurashima. (2003) WO3 Nanorods/Nanobelts Synthesized via Physical Vapor Deposition Process [J]. Chemical Physics Letters, 367(1), 214-218.

[22] Y. He, J.-A. Wang, W.-B. Sang, R.-F. Wu, L.-L. Yan and Y.-Y. Fang. (2005) Self-assembling Generated via Polymer and the Formation Mechanism [J]. Act. Chimiea Sinica, 63(12), 1037-1041. 